\title{
Transforaminal Percutaneous Endoscopic Discectomy for Lumbar Disc Herniation in Parkinson's Disease: A Case-Control Study
}

\author{
Stylianos Kapetanakis ${ }^{1}$, Eirini Giovannopoulou ${ }^{1}$, George Charitoudis ${ }^{1}$, Konstantinos Kazakos ${ }^{2}$ \\ ${ }^{1}$ Spine Department and Deformities, Interbalkan European Medical Center, Thessaloniki, Greece \\ ${ }^{2}$ Department of Orthopedic Surgery, University Hospital of Alexandroupolis, Medical School, Democritus University of Thrace, Alexandroupolis, Greece
}

Study Design: A case-control study.

Purpose: To investigate the effectiveness of transforaminal percutaneous endoscopic discectomy (TPED) in Parkinson's disease (PD). Overview of Literature: Patients with PD frequently suffer from radiculopathy and low back pain. Additionally, they demonstrate higher complication rates after open spine surgery. However, the clinical outcome of minimally invasive techniques for lumbar discectomy, such as TPED, have not been established for this population.

Methods: Patients diagnosed with lumbar disc hernia were divided into Group A (11 patients diagnosed with PD), and Group B (10 patients as the control, non-PD group). All patients underwent TPED. Indexes of visual analogue scale (VAS) for leg pain and Oswestry disability index (ODI) were assessed right before surgery and at six weeks, three months, six months and one year post-surgery.

Results: At the baseline visit, groups did not differ significantly with age $(p=0.724)$, gender $(p=0.835)$, level of operation $(p=0.407)$, ODI ( $p=0.497)$ and VAS ( $p=0.772)$. Parkinson's patients had higher scores in ODI at every visit, but the outcome was statistically significant only at 3 months $(p=0.004)$ and one year $(p=0.007)$. Similarly, VAS measurements were higher at each time point, with the difference being significant at $3(p<0.001), 6(0.021)$, and $12(p<0.001)$ months after surgery. At the end of a year of follow up, ODI was reduced by $49.6 \%( \pm 16.7)$ in Group $A$ and $59.2 \%( \pm 8.0)$ in Group B $(p=0.111)$, translating to a $79.5 \%( \pm 13.0)$ and $91.5 \%( \pm 4.1)$ average improvement in daily functionality ( $p=0.024)$. VAS was reduced by $59.1 \mathrm{~mm}( \pm 11.8)$ in Group $A$ and $62.2 \mathrm{~mm}( \pm 7.4)$ in Group $B(p=0.485)$, leading to an $85.3 \%( \pm 4.0)$ and $91.9 \%( \pm 2.6)$ general improvement in leg pain $(p<0.001)$.

Conclusions: Our data indicate that TPED led to satisfactory improvement in leg pain and daily living in PD patients a year after surgery.

Keywords: Lumbar spine; Parkinson disease; Percutaneous discectomy; Endoscopy

\section{Introduction}

Intervertebral disc pathology is a common problem in patients suffering from Parkinson's Disease (PD). PD is a degenerative disorder of the central nervous system affecting the basal ganglions with an incidence of 17 per 100,000 of population per year. The disease is diagnosed in the sixth decade of life with a peak incidence between 70 and 79 years of age [1]. PD population is generally characterized by older age, neuromuscular impairment,

Received Dec 7, 2015; Revised Jan 15, 2016; Accepted Jan 19, 2016

Corresponding author: Stylianos Kapetanakis

Spine Department and Deformities, Interbalkan European Medical Center, Thessaloniki, PC 57001, Greece

Tel: +30-69-7270-7384, Fax: +30-23-1128-9109, E-mail: stkapetanakis@yahoo.gr 
poor bone quality, higher incidence of back pain and radiculopathy [2]. Moreover, the onset of the disease at the sixth decade coincides with profound degeneration of the intervertebral discs due to aging.

Lumbar discectomy is the most frequently performed spinal surgery [3]. Traditional open discectomy is replaced by less invasive surgical techniques for disc excision [4]. Microdiscectomy is now the gold standard [5]. Endoscopy, however, has become popular among the spinal surgeons in the last 30 years and can be performed with a posterior or posterolateral approach [6]. For transforaminal percutaneous endoscopic discectomy (TPED), the approach is to the epidural space through the intervertebral foramen. The technique combines the benefits of endoscopy (direct visualization, reduced traumatization, hospital stay and postsurgical morbidity [7]) with optimal exposure of the intervertebral space and preservation of the dorsal musculature, the vertebrae and the ligamentous structures $[8,9]$.

Open procedures are accompanied with high complication rates and high recurrence rates in the $\mathrm{PD}$ population, and instability is the prevalent mechanism [10]. PD results in spinal instability due to muscular rigidity, stooped posture of the trunk and paraspinal muscles myopathy [11]. However, via the transforaminal endoscopic approach, the lamina, the ligaments and the dorsal musculature are preserved and the spine is not further destabilized.

To our knowledge, no previous study exists in the literature on the clinical outcome of TPED in PD patients diagnosed with lumbar disc herniation. In this study, we intended to (A) examine the progress of pain a year after TPED in PD patients and (B) compare the results with healthy individuals.

\section{Materials and Methods}

\section{Patients and their baseline demographics}

All patients agreed to participate in the study and signed an informed written consent. The study was approved by the medical council of the hospital and the local ethics committee. All patients of our study were diagnosed with lumbar disc hernia and fulfilled the indications required for discectomy.

Inclusion criteria were: (1) radiculopathy, (2) positive nerve root tension sign, (3) sensory or motor neurologic lesion on clinical examination, (4) hernia confirmed by magnetic resonance imaging (MRI) of the lumbar spine and in compliance with clinical findings, and (5) failure of a 12-week conservative treatment.

Exclusion criteria were: (1) non-contaminated disc hernia exceeding one third of the spinal canal on the sagittal MRI scans, (2) sequestration of the disc, (3) central or lateral recess spinal stenosis, (4) recurrent herniated disc or previous surgery at the affected level, (5) segmental instability or spondylolisthesis, (6) spinal tumor or infection, and (7) vertebral fracture.

Patients were divided in two groups according to the diagnosis of PD. Group A consisted of 11 patients with PD (52.4\%), while Group B consisted of 10 healthy individuals (47.6\%). Baseline characteristics are shown at Table 1. The two groups did not have any statistical significant differences in age, gender, level of operation, ODI and VAS measures pre-surgery.

\section{Methods}

All patients were scheduled to undergo TPED at the same center by the same experienced surgeon. Outcome was measured by VAS for leg pain and the self-administrated Oswestry low back pain disability questionnaire. Patients were asked to complete the measurements right before surgery. The same procedure was repeated following the surgery at 6 weeks, 3 months, 6 months, and one year. Our primary hypothesis was that reduction or progression of leg pain (measured with VAS) and daily life disability (from the ODI) would not significantly differ between patients with PD and healthy individuals a year after TPED.

\section{Surgical technique}

The surgery was TPED. The procedure was performed under local anesthesia and mild sedation. All patients were monitored in terms of blood pressure, pulse rate, oxygen saturation and electrocardiographic signals. Patients were positioned at the lateral decubitus position, lying down on the opposite site for the lesion to face upwards. After the disinfection of the surgical field, local anesthesia was initially performed at the needle entry site. The needle was placed through the Kambin's triangle [12], $11 \mathrm{~cm}$ of the midline, and under the fluoroscopic technique. After verification of the level, mild sedation and analgesia were provided with fentanyl (fentanyl ampule), as the enlargement of the neural foramen is painful. The compliance of 
Table 1. Patients' demographic characteristics in group $A$ and $B$

\begin{tabular}{lccc} 
Characteristic & Group A & Group B & $p$-value \\
\hline $\begin{array}{l}\text { Patients } \\
\text { Sex }\end{array}$ & 11 & 10 & - \\
\hline Male & $6(54.5)$ & $5(50.0)$ & 0.835 \\
\hline Female & $5(45.4)$ & $5(50.0)$ & \\
\hline Level & & & 0.407 \\
\hline L3-L4 & $3(27.3)$ & $2(20.0)$ & \\
\hline L4-L5 & $4(36.4)$ & $5(50.0)$ & \\
\hline L5-S1 & $4(36.4)$ & $3(30.0)$ & 0.724 \\
\hline Age (yr) & $62.9( \pm 6.0)$ & $61.8( \pm 8,1)$ & 0.497 \\
\hline ODI preoperatively (\%) & $61.2( \pm 13.8)$ & $64.6( \pm 7.4)$ & 0.772 \\
\hline VAS preoperatively $(\mathrm{mm})$ & $69.0( \pm 11.7)$ & $67.7( \pm 8.0)$ & \\
\hline
\end{tabular}

Values are presented as number (\%) or mean \pm standard deviation.

ODI, Oswestry disability index; VAS, visual analogue scale.

the patients was affected during the sequential passage of 3 different size reamers (5.5, 6.5, 7.5 mm, Joymax System). The cannula and the endoscope were then placed and the nerve root was secured. Subsequently, the discectomy was performed with graspers. The patients were monitored for the following hour in the wards and then mobilized. The patients were discharged one day after surgery. Perioperative complications were not observed.

\section{Visual analogue scale for leg pain}

Visual analogue scales (VAS) have been introduced early in the 1920 's, in order to quantitate a wide range of subjective clinical phenomena, including pain [13]. We used a unipolar, horizontal line of $100 \mathrm{~mm}$ length. The patients were asked to point with a mark the subjective intensity of their leg pain on the line. Finally, the personal score was measured in millimeters $(\mathrm{mm})$, giving an objective assessment of the patient's radicular symptoms. Minimal clinically significant change is regarded as at least a $9 \mathrm{~mm}$ change in VAS, independent of gender, age, and pain etiology [14].

\section{Oswestry disability index}

Oswestry disability index (ODI) is a self-administrated questionnaire, specified in the evaluation of low back pain in daily living. First introduced in 1980 by Fairbank et al. [15], it has now evolved to the main outcome measure in the clinical evaluation of spinal disorders and effectiveness of spinal surgery [16], as it is easy and reliable [17]. Patients were asked to select the statement that best described their condition. The total score comprises the percentage of disability in their day living due to low back pain. The minimal clinically evident improvement is associated with a reduction by at least $10 \%$ in ODI [17].

\section{Statistical analysis}

Continuous variables (age, ODI, VAS) are expressed as mean \pm standard deviation $(\mathrm{SD})$ and categorical variables (gender, level of operation) as percentages. The KolmogorovSmirnov test was utilized for normality analysis. Pearson (or chi-square test) was used for the analysis of qualitative and categorical variables. Student's $t$-test and MannWhitney $U$-test were for quantitative and continuous variables, and for normality of distributions, respectively.

ODI and VAS measures were re-assessed at 6 weeks, 3 months, 6 months and a year after the TPED. We looked for any significant differences between the groups for the ODI and VAS indexes, as well as for absolute changes and percentage changes at the follow-up intervals postsurgery.

\section{Results}

At baseline visit, ODI ( $p=0.497)$ and VAS $(p=0.772)$ scores did not differ significantly between groups. Mean values 
Table 2. ODI values preoperatively and at 6 weeks, 3 months, 6 months, and 12 months after surgery

\begin{tabular}{lcccc} 
Variable & Total population & Group A & Group B & $p$-value ${ }^{\text {a) }}$ \\
ODI preoperatively & $69.9( \pm 11.0)$ & $61.2( \pm 13.8)$ & $64.6( \pm 7.4)$ & 0.497 \\
ODI 6 wk postoperatively & $19.3( \pm 5.0)$ & $20.7( \pm 5.7)$ & $17.8( \pm 3.8)$ & 0.190 \\
\hline ODI 3 mo postoperatively & $12.1( \pm 4.2)$ & $14.5( \pm 3.9)$ & $9.4( \pm 2.7)$ & $\mathbf{0 . 0 0 4}$ \\
ODI 6 mo postoperatively & $9.1( \pm 2.9)$ & $10.0( \pm 3.1)$ & $8.2( \pm 2.4)$ & 0.085 \\
ODI 12 mo postoperatively & $6.15( \pm 5.5)$ & $11.6( \pm 6.0)$ & $5.4( \pm 2.3)$ & $\mathbf{0 . 0 0 7}$ \\
\hline
\end{tabular}

Values are presented as mean \pm standard deviation.

ODI, Oswestry disability index; SD, standard deviation.

${ }^{\text {a) }} p$-values considered to be important are in bold. Level of significance $a=0.050$.

Table 3. VAS values preoperatively and at 6 weeks, 3 months, 6 months, and 12 months after surgery

\begin{tabular}{lcccc} 
Variable & Total population & Group A & Group B & $p$-value \\
VAS preoperatively & $63.38( \pm 9.9)$ & $69.0( \pm 11.7)$ & $67.7( \pm 8.0)$ & 0.772 \\
VAS 6 wk postoperatively & $29.71( \pm 7.0)$ & $32.3( \pm 7.8)$ & $26.9( \pm 5.0)$ & 0.079 \\
VAS 3 mo postoperatively & $14.42( \pm 5.4)$ & $20.4( \pm 4.5)$ & $13.0( \pm 3.2)$ & $8.7( \pm 3.6)$ \\
VAS 6 mo postoperatively & $8.9( \pm 3.2)$ & $11.9( \pm 2.0)$ & $5.5( \pm 2.0)$ & $<.001$ \\
VAS 12 mo postoperatively & $7.8( \pm 3.0)$ & $9.9( \pm 2.0)$ & $<0.001$ \\
\hline
\end{tabular}

VAS, visual analogue scale.

Table 4. Absolute interval reduction of ODI (\%) and VAS (mm)

\begin{tabular}{|c|c|c|c|}
\hline Time intervals & Group A & Group B & $p$-value ${ }^{\text {a) }}$ \\
\hline \multicolumn{4}{|c|}{ Absolute reduction of ODI (\%) } \\
\hline ODI preop.-6 wk & $40.6( \pm 14.5)$ & $46.8( \pm 6.3)$ & 0.214 \\
\hline ODI 6 wk-3 mo & $6.2( \pm 4.2)$ & $8.4( \pm 3.1)$ & 0.191 \\
\hline ODI 3 mo-6 mo & $4.6( \pm 4.3)$ & $1.2( \pm 2.7)$ & 0.061 \\
\hline ODI 6 mo-12 mo & $-0.2( \pm 5.0)$ & $2.8( \pm 2.8)$ & 0.029 \\
\hline ODI preop.-12 mo & $49.6( \pm 16.7)$ & $59.2( \pm 8.0)$ & 0.111 \\
\hline \multicolumn{4}{|c|}{ Absolute reduction of VAS (mm) } \\
\hline VAS preop. -6 wk & $36.7( \pm 7.3)$ & $40.8( \pm 6.4)$ & 0.314 \\
\hline VAS 6 wk-3 mo & $11.9( \pm 8.4)$ & $13.9( \pm 5.2)$ & 0.519 \\
\hline VAS 3 mo-6 mo & $8.6( \pm 3.9)$ & $4.3( \pm 4.1)$ & 0.023 \\
\hline VAS 6 mo-12 mo & $1.9( \pm 2.6)$ & $3.2( \pm 3.0)$ & 0.349 \\
\hline VAS preop.-12 mo & $59.1( \pm 11.8)$ & $62.2( \pm 7.4)$ & 0.485 \\
\hline
\end{tabular}

ODI, Oswestry disability index; VAS, visual analogue scale; preop., preoperatively.

a) $p$-values considered to be important are in bold. Level of significance $a=0.050$.

for both groups were $69.9 \%( \pm 11.0)$ for ODI and 63.38 $\mathrm{mm}( \pm 9.9)$ for VAS.

ODI (\%) was significantly higher in Group A only at 3 months $(14.5 \pm 3.9$ vs. $9.4 \pm 2.7, p=0.004)$ and at 12 months (11.6 \pm 6.0 vs. $5.4 \pm 2.3, p=0.007$ ) after surgery (Table 2 ).

VAS ( $\mathrm{mm})$ was higher in Group A at every interval, although this difference was significant at 3 months (20.4 \pm 4.5 vs. $13.0 \pm 3.2, p<0.001), 6$ months ( $11.9 \pm 2.0$ vs. 8.7 $\pm 3.6, p=0.021)$ and 12 months $(9.9 \pm 2.0$ vs. $5.5 \pm 2.0$, $p<0.001$ ) (Table 3 ).

The progress of outcome was assessed by (1) the absolute reduction (Table 4 ) and (2) the percentage im- 
Table 5. Percentage interval improvement of ODI and VAS

\begin{tabular}{|lccc}
\multicolumn{1}{c}{ Time intervals } & Group A & Group B & $p$-value ${ }^{\text {a) }}$ \\
\hline Improvement of ODI (\%) & & & \\
\hline ODI preop.-6 wk & $64.8( \pm 10.8)$ & $72.5( \pm 4.9)$ & $\mathbf{0 . 0 2 0}$ \\
\hline ODI 6 wk-3 mo & $27.4( \pm 16.4)$ & $46.6( \pm 13.0)$ & $\mathbf{0 . 0 0 8}$ \\
\hline ODI 3 mo-6 mo & $28.6( \pm 27.3)$ & $9.5( \pm 24.6)$ & 0.114 \\
\hline ODI 6 mo-12 mo & $-15.9( \pm 47.2)$ & $29.5( \pm 29.1)$ & $\mathbf{0 . 0 2 0}$ \\
\hline ODI preop.-12 mo & $79.5( \pm 13.0)$ & $91.5( \pm 4.1)$ & $\mathbf{0 . 0 2 4}$ \\
\hline Improvement of VAS (\%) & & $60.2( \pm 6.0)$ & $\mathbf{0 . 0 3 2}$ \\
\hline VAS preop.-6 wk & $53.4( \pm 7.3)$ & $50.6( \pm 13.1)$ & $\mathbf{0 . 0 3 3}$ \\
\hline VAS 6 wk-3 mo & $33.6( \pm 19.7)$ & $31.3( \pm 28.5)$ & 0.353 \\
\hline VAS 3 mo-6 mo & $40.3( \pm 12.6)$ & $31.6( \pm 23.1)$ & 0.082 \\
\hline VAS 6 mo-12 mo & $14.3( \pm 20.1)$ & $91.9( \pm 2.6)$ & $<\mathbf{0 . 0 0 1}$ \\
\hline VAS preop.-12 mo & $85.3( \pm 4.0)$ & \\
\hline
\end{tabular}

preop., preoperatively; ODI, Oswestry disability index; VAS, visual analogue scale.

${ }^{\text {a) }} p$-values considered to be important are in bold. Level of significance $a=0.050$.

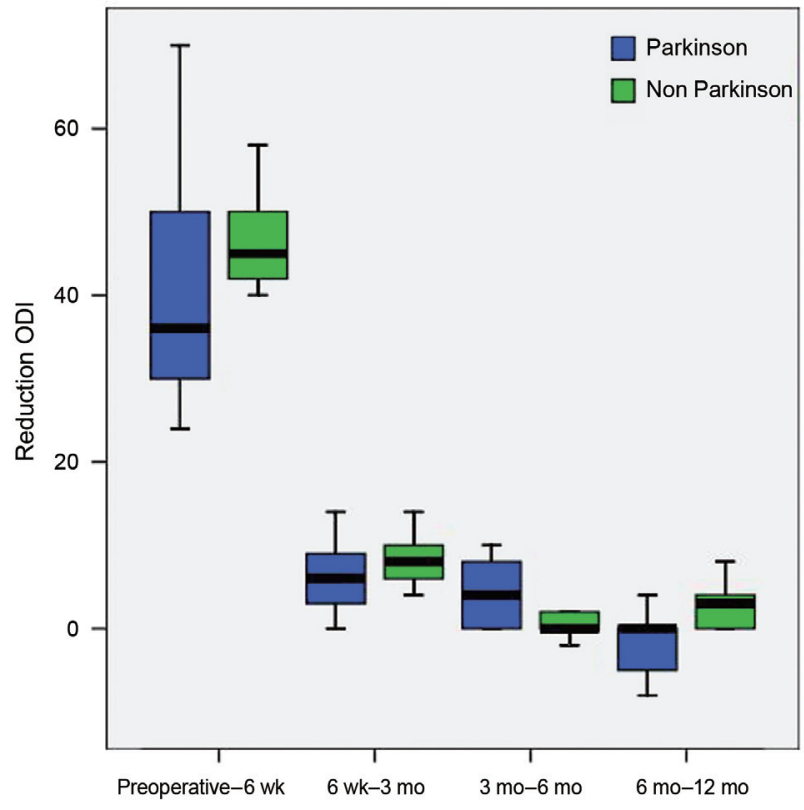

Fig. 1. Comparative progress of ODI in Parkinson's and non-Parkinson's patients. ODI, Oswestry disability index.

provement (Table 5) of ODI and VAS between each time interval (6 weeks, 3 months, 6 months, and 1 year) after surgery. The comparative progress is demonstrated in Figs. 1 and 2.

At the end of a year of follow-up, ODI was reduced by $49.6 \%( \pm 16.7)$ in Group A and 59.2\% $( \pm 8.0)$ in Group B $(p=0.111)$, which is interpreted as $79.5 \%( \pm 13.0)$ and

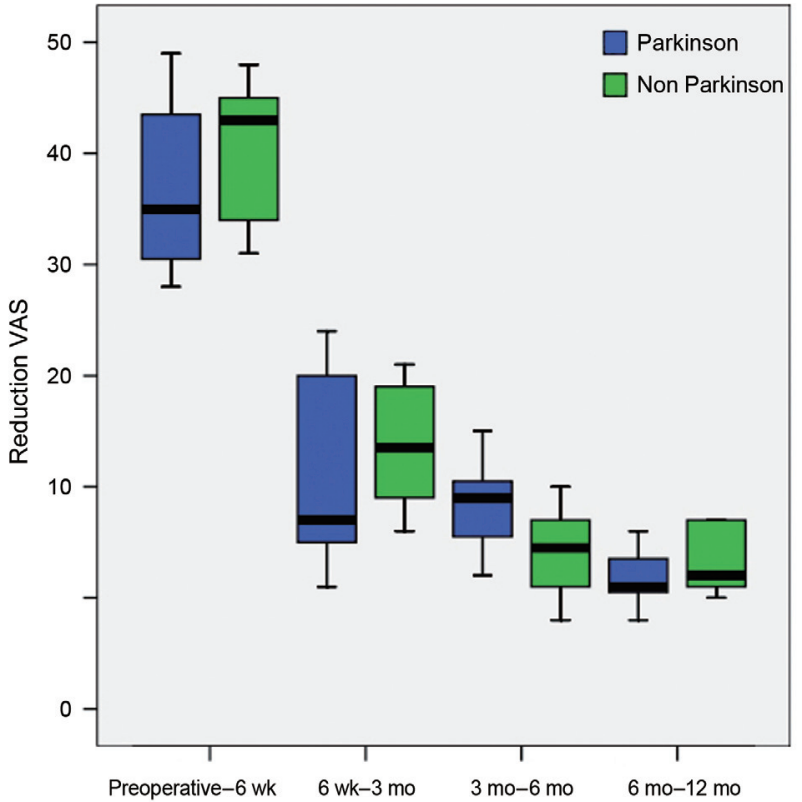

Fig. 2. Comparative progress of VAS in Parkinson's and non-Parkinson's patients. VAS, visual analogue scale.

$91.5 \%( \pm 4.1)$, respectively, average improvement in daily functionality $(p=0.024)$. Accordingly, VAS was reduced by $59.1 \mathrm{~mm}( \pm 11.8)$ in Group A and $62.2 \mathrm{~mm}( \pm 7.4)$ in Group B ( $p=0.485)$, meaning general improvement for leg pain of $85.3 \%( \pm 4.0)$ and $91.9 \%( \pm 2.6)$, respectively $(p<0.001)$. 


\section{Discussion}

PD induces several neuromuscular changes affecting the biomechanics of a patient's spine. Postural deformities are common clinical presentations of $\mathrm{PD}$, and they may be detected at the sagittal and/or the coronal plane. Pathophysiology of those changes is multifactorial. Postural spine imbalance may be the outcome of central nervous system changes that induce flexion dystonia of the trunk, muscle rigidity and impaired proprioception and kinaesthesia. Administrated drugs such as dopamine agonists are also potential deteriorating factors. Peripheral mechanisms, such as localized myopathy of the back muscles, act complementary to the establishment of the spine deformity. Soft tissue changes in PD patients are the result of previous spine surgery or aging. It is not yet known whether these changes lead to disordered trunk positioning or result from it [18]. In either case, altered spine biomechanics and abnormal force distributions stress the lumbar vertebra, the discs and the paraspinal soft tissues, accelerating disc degeneration and hernia formation [2].

Following spine surgery, a neuromuscular disorder and induced spinal deformity tend to increase complications [19-22]. Therefore, the PD population is subjected to higher incidence of complications after spinal surgery. Predominant causes are excessive lumbar kyphosis, the adjacent level instability in cases of interbody fusion and equipment-related complications such as hardware failure or displacement [10]. Extended resection of the lamina is shown to negatively affect stability at the operated level [23]. Laminectomy accompanied by interbody fusion, especially when it is multilevel and does not achieve ideal alignment on sagittal and coronal plane, may deteriorate spinal imbalance in PD patients, since physiological adjustment mechanisms to compensate for altered biomechanics are weak. Moreover, flexed posture of the trunk increases the stress on instrumentation, especially for the L5-S1 level [11]. PD poses specific limitations on open spinal surgery, and taking into account that instability is mainly associated with the resection of the dorsal stabilizing structures (lamina, ligaments, muscles) rather that the discectomy itself [23], minimally invasive surgery could be an alternative for those patients.

To our knowledge, no existing study has investigated the effectiveness of TPED for the management of lumbar disc hernia in PD patients. As TPED preserves the biomechanics of the lumbar spine (soft tissues and bony struc- tures) and offers stability to the operated and adjacent levels due to the smaller traumatization of the supportive paraspinal tissues, TPED is theoretically associated with many advantages in those patients.

VAS and ODI are commonly used instruments to assess clinical progress after spinal surgery. PD patients are expected to have a higher prevalence of pain and radiculopathy [2], a fact that explains the higher values in ODI and VAS scales in PD patients found in our study. The difference, though, becomes significant only at 3 and 12 months for ODI and at 3, 6, and 12 months for VAS. These periodical differences are not assessed, as they were below the limit of minimum clinical significance. The interval reductions in VAS and ODI were statistically significantly lower in PD cases only between 3 and 6 months and between 6 and 12 months, respectively, but without clinical impact as changes $\leq 10 \%$ for OD and $\leq 9 \mathrm{~mm}$ for VAS are not consistent with clinically detectable improvements $[14,17]$.

According to a systematic review of literature, TPED seems to induce a mean of $88 \%$ reduction in the VAS scale for leg pain, with a range of $65 \%$ to $89 \%$ reduction according to different studies. Improvement of functionality in daily activities follows the recession of leg pain after surgery. Specifically, in literature ODI improved by a mean of $83 \%$, with a range of $74 \%$ and $90 \%$ [24]. The studies had follow-up periods ranging from 6 weeks to 48 months. In our study, absolute reductions in VAS and ODI showed no statistical differences between the two groups a year after TPED. VAS and ODI improvement, however, was significantly lower in the PD population, but was still within the expected range for the normal population. This inconsistency between absolute and percentage changes could be attributed to the higher preoperative values in the PD population and the small number of patients in the study. Moreover, ODI was stabilized after the first month, while recession of leg pain (VAS) continued until the third month post-surgery in both groups. Mean interval changes after this crucial period were beyond clinical significance.

\section{Conclusions}

Initial results from our study suggest that TPED may be a good alternative in PD patients diagnosed with lumbar disc hernia, as recession of leg pain and improvement of everyday life were satisfactory a year after the procedure. 


\section{Conflict of Interest}

No potential conflict of interest relevant to this article was reported.

\section{References}

1. Twelves D, Perkins KS, Counsell C. Systematic review of incidence studies of Parkinson's disease. Mov Disord 2003;18:19-31.

2. Broetz D, Eichner M, Gasser T, Weller M, Steinbach JP. Radicular and nonradicular back pain in Parkinson's disease: a controlled study. Mov Disord 2007;22: 853-6.

3. Weinstein JN, Tosteson TD, Lurie JD, et al. Surgical vs nonoperative treatment for lumbar disk herniation: the Spine Patient Outcomes Research Trial (SPORT): a randomized trial. JAMA 2006;296:244150.

4. Mixter WJ, Barr JS. Rupture of the intervertebral disc with involvement of the spinal canal. N Engl J Med 1934;211:210-5.

5. Postacchini F, Postacchini R. Operative management of lumbar disc herniation: the evolution of knowledge and surgical techniques in the last century. Acta Neurochir Suppl 2011;108:17-21.

6. Brayda-Bruno M, Cinnella P. Posterior endoscopic discectomy (and other procedures). Eur Spine J 2000; 9 Suppl 1:S24-9.

7. Mayer HM, Brock M. Percutaneous endoscopic lumbar discectomy (PELD). Neurosurg Rev 1993;16:11520.

8. Mathews HH. Transforaminal endoscopic microdiscectomy. Neurosurg Clin N Am 1996;7:59-63.

9. Yeung AT, Yeung CA. Minimally invasive techniques for the management of lumbar disc herniation. Orthop Clin North Am 2007;38:363-72.

10. Babat LB, McLain RF, Bingaman W, Kalfas I, Young P, Rufo-Smith C. Spinal surgery in patients with Parkinson's disease: construct failure and progressive deformity. Spine (Phila Pa 1976) 2004;29:2006-12.

11. Koller H, Acosta F, Zenner J, et al. Spinal surgery in patients with Parkinson's disease: experiences with the challenges posed by sagittal imbalance and the Parkinson's spine. Eur Spine J 2010;19:1785-94.

12. Kambin P, Gellman H. Percutaneous lateral discec- tomy of the lumbar spine: a preliminary report. Clin Orthop 1983;174:127-32.

13. Wewers ME, Lowe NK. A critical review of visual analogue scales in the measurement of clinical phenomena. Res Nurs Health 1990;13:227-36.

14. Kelly AM. Does the clinically significant difference in visual analog scale pain scores vary with gender, age, or cause of pain? Acad Emerg Med 1998;5:1086-90.

15. Fairbank JC, Couper J, Davies JB, O'Brien JP. The Oswestry low back pain disability questionnaire. Physiotherapy 1980;66:271-3.

16. Fairbank JC, Pynsent PB. The Oswestry Disability Index. Spine (Phila Pa 1976) 2000;25:2940-52.

17. Davidson M, Keating JL. A comparison of five low back disability questionnaires: reliability and responsiveness. Phys Ther 2002;82:8-24.

18. Doherty KM, van de Warrenburg BP, Peralta MC, et al. Postural deformities in Parkinson's disease. Lancet Neurol 2011;10:538-49.

19. Gau YL, Lonstein JE, Winter RB, Koop S, Denis F. Luque-Galveston procedure for correction and stabilization of neuromuscular scoliosis and pelvic obliquity: a review of 68 patients. J Spinal Disord 1991;4: 399-410.

20. Bell DF, Moseley CF, Koreska J. Unit rod segmental spinal instrumentation in the management of patients with progressive neuromuscular spinal deformity. Spine (Phila Pa 1976) 1989;14:1301-7.

21. Banta JV, Drummond DS, Ferguson RL. The treatment of neuromuscular scoliosis. Instr Course Lect 1999;48:551-62.

22. Swank SM, Cohen DS, Brown JC. Spine fusion in cerebral palsy with L-rod segmental spinal instrumentation: a comparison of single and two-stage combined approach with Zielke instrumentation. Spine (Phila Pa 1976) 1989;14:750-9.

23. Iida $\mathrm{Y}$, Kataoka O, Sho T, et al. Postoperative lumbar spinal instability occurring or progressing secondary to laminectomy. Spine (Phila Pa 1976) 1990;15:11869.

24. Nellensteijn J, Ostelo R, Bartels R, Peul W, van Royen B, van Tulder M. Transforaminal endoscopic surgery for symptomatic lumbar disc herniations: a systematic review of the literature. Eur Spine J 2010;19:181204. 